REVISTA

EPUEACIÓN
Revista Educación

ISSN: 0379-7082

ISSN: 2215-2644

revedu@gmail.com

Universidad de Costa Rica

Costa Rica

\title{
Relación entre el uso del aula virtual y el rendimiento académico en estudiantes del curso de Bioquímica para Enfermería de la Universidad de Costa Rica
}

\author{
Granados-Zúñiga, Jorge \\ Relación entre el uso del aula virtual y el rendimiento académico en estudiantes del curso de Bioquímica \\ para Enfermería de la Universidad de Costa Rica \\ Revista Educación, vol. 43, núm. 2, 2019 \\ Universidad de Costa Rica, Costa Rica \\ Disponible en: http://www.redalyc.org/articulo.oa?id=44058158027 \\ DOI: https://doi.org/10.15517/revedu.v43i2.32723
}

Esta obra está bajo una Licencia Creative Commons Atribución-NoComercial-SinDerivar 3.0 Internacional. 


\title{
Relación entre el uso del aula virtual y el rendimiento académico en estudiantes del curso de Bioquímica para Enfermería de la Universidad de Costa Rica
}

\author{
The Relationship between Virtual Classrooms and Academic Performance among Nursing Students in a \\ University of Costa Rica Biochemistry Course \\ Jorge Granados-Zúñiga \\ Escuela de Medicina, Universidad de Costa Rica, Costa \\ Rica \\ jorge.granados@ucr.ac.cr \\ (iD http://orcid.org/0000-0002-4197-0404 \\ DOI: https://doi.org/10.15517/revedu.v43i2.32723 \\ Redalyc: http://www.redalyc.org/articulo.oa?id=44058158027
}

Recepción: 14 Abril 2018

Aprobación: 03 Junio 2019

\section{Resumen:}

El objetivo de este estudio fue investigar la relación entre el uso del aula virtual y el rendimiento académico en el curso de Bioquímica para Enfermería que imparte la Escuela de Medicina de la Universidad de Costa Rica (UCR). Un primer análisis fue de carácter retrospectivo mediante la revisión de las actas de notas de los cursos impartidos durante el período 2000-2017. No se observó una diferencia estadísticamente significativa (prueba t-Student, $\alpha=0.05$ ) en el porcentaje de aprobación del curso entre antes (70 \%) y después (59.5 \%) del año 2009, fecha en la que se comenzó a utilizar el aula virtual. Un segundo análisis fue de carácter transversal mediante la correlación entre la nota obtenida en las evaluaciones y el uso del aula virtual por cada estudiante $(\mathrm{n}=55)$ en el curso ofrecido durante el segundo ciclo lectivo de 2017. Para el primer examen de dicho curso no se observó una correlación estadísticamente significativa entre la nota y la cantidad de veces que cada estudiante utilizó el aula virtual (prueba t-pareada, $\alpha=$ 0.05); para las evaluaciones siguientes la correlación se incrementó, pero fue baja. Finalmente, se discuten las posibles razones del aparente bajo impacto que el uso del aula virtual ha tenido en este curso y la conveniencia de replantearse la estrategia pedagógica incrementando las lecturas lineales de textos impresos y las actividades docentes en el aula.

Palabras Clave: Rendimiento académico, TIC, Tecnología educativa, Aulas virtuales, Educación superior.

\section{Abstract:}

This study examines the relationship between the use of virtual classrooms and student academic performance as analyzed in a Biochemistry course for nursing students at the University of Costa Rica (UCR) Medical School. An initial retrospective study which reviewed the course minutes recorded from 2000-2017 was done. No statistically significant differences were observed ( $t$ test, $\alpha=0.05)$ in the percentage of students who passed the course prior to 2009 (70\% passing rate) versus afterwards $(59.5 \%$ passing rate) with the use of virtual classrooms. A cross-cutting analysis was then conducted which assessed the correlation between student grades for each exam after they used the virtual classroom $(n=55)$ during the second semester of 2017. No statistically significant correlations were observed with regards to student grades and frequency of virtual classroom visits (paired $t$-test, $\alpha=$ 0.05). Subsequent testing did, however, reveal a slight grade increase. Finally, the possible reasons for minimal impact of the virtual classroom on this course are discussed in addition to reconsidering pedagogical strategies by increasing printed text linear reading and teaching activities in the classroom.

KEYWORDS: Academic achievement, ICT, Teaching technology, Virtual classrooms, Higher education.

\section{INTRODUCCIÓN}

El concepto de tecnología educativa se refiere al diseño de estrategias, uso de medios y control de sistemas de comunicación para la enseñanza, y emergió vinculado al uso en educación de los medios audiovisuales modernos, según lo recogió la UNESCO en 1984 (Luján y Salas, 2009). Es “un proceso complejo e integrado que incluye personas, procedimientos, ideas, aparatos y organizaciones para analizar problemas y proyectar, 
aplicar, evaluar y administrar soluciones a estos problemas relacionados con todos los aspectos del aprendizaje humano" (Luján y Salas, 2009, p. 9).

Las tecnologías de la información y la comunicación [TIC] y las plataformas virtuales como apoyo o alternativa para la educación presencial son tecnologías educativas con el fin de mejorar el aprendizaje y el rendimiento académico. El uso de recursos tecnológicos en la educación superior parte de premisas optimistas en torno a que estos logran estímulos auditivos y visuales que propician el aprendizaje y la reconstrucción del conocimiento desarrollando nuevas experiencias docentes, y habilidades y destrezas en docentes y estudiantes (Regueyra, 2011).

Sin embargo, la valoración que el efecto de estos recursos posee sobre el rendimiento académico o el aprendizaje se desarrolla, usualmente, a partir de consultas de la opinión de estudiantes y docentes, lo cual puede resultar subjetivo e impreciso. Por ejemplo, en un sondeo con docentes de universidades privadas en Guatemala (Rivera, Fernández, Guzmán, y Eduardo, 2017) se evidencia que aquellos son conscientes de la importancia de las TIC y perciben su utilidad en términos de un aumento en el rendimiento del usuario o el aprovechamiento que obtiene de las tareas que efectúa. En otro caso, la Universidad Centroccidental Lisandro Alvarado de Venezuela ofreció un curso semipresencial por medio del aula virtual para la enseñanza de la matemática en ingeniería como una manera de responder a la sobredemanda de esta asignatura (Troncoso, Cuicas y Debel, 2010). Los autores señalan que el aula virtual fue eficaz, puesto que el número de estudiantes aprobados es superior al de aplazados. Sin embargo, cabe cuestionarse si este es un criterio adecuado para determinar el rendimiento académico, pues las categorías aprobado o aplazado podrían estar subvalorando diferencias relevantes en este aspecto. Además, no se aplicaron pruebas de significancia estadística ni de hipótesis para determinar si los dos grupos que participaron en el curso bimodal, de un total de 7 , obtuvieron una promoción significativamente mejor.

Tomando en cuenta el amplio uso de las tecnologías educativas y lo poco que se ha investigado acerca del efecto que tienen sobre el rendimiento académico en Costa Rica, se ha propuesto la presente investigación. $\mathrm{Al}$ respecto cabe preguntarse no solo el cómo y por qué un medio opera en el aprendizaje, sino por qué se puede utilizar (Luján y Salas, 2009). De ahí que el objetivo de esta investigación fue evaluar la relación que existe entre el uso de plataformas virtuales y el rendimiento académico en el curso de Bioquímica para Enfermería que imparte la Escuela de Medicina de la Universidad de Costa Rica (UCR). La pesquisa siguió dos estrategias: en primer lugar, se analizó el porcentaje de aprobación del curso a lo largo de un período de 18 años, para intentar establecer la repercusión que tuvo sobre este la incorporación del aula virtual y, en segundo lugar, se estudió el rendimiento académico en un grupo de estudiantes durante un ciclo lectivo para establecer si existe correlación entre las notas obtenidas a lo largo del curso y el uso del aula virtual.

\section{AnTECEDENTES}

Hace quince años en Costa Rica se preveía que la utilización de lo que entonces eran las nuevas TIC en la educación representaría una transformación de las relaciones interpersonales y nuevas formas de percibir el tiempo y el espacio (Gurdián, 2001). A principios del presente siglo, un análisis general de los desafíos de la educación superior costarricense señalaba que la incorporación de la tecnología educativa debería permitir modernizar, flexibilizar y dinamizar los procesos de enseñanza y aprendizaje significativo y sacarlo del enfoque tradicional conductista (Hernández, 2002).

Para algunos autores dicha tecnología educativa ha quedado enlazada con el aprendizaje a partir de la propuesta de la instrucción programada, desarrollada desde el enfoque conductista (Salas, 2002). En su momento se apoyó el uso de la tecnología educativa señalando su capacidad para responder a las nuevas exigencias que plantean las concepciones modernas sobre la enseñanza y el aprendizaje desde el constructivismo, pero haciendo la salvedad de que no puede insertarse y actuar por sí misma, sino que opera a partir de la inteligencia y la creatividad humanas (Hernández, 2002). 
En medio de estas concepciones ha existido tanto una visión optimista de la informática educativa (según la cual el educador ocupará un lugar sobresaliente como estimulador de la creatividad del estudiante) como una visión pesimista (en la cual el personal docente será desplazado por la máquina). También, la preeminencia de la computadora y la informática, y el riesgo de que las sociedades que no asuman una actitud positiva frente a esta tecnología sean condenadas al subdesarrollo (Carvajal, 2002).

Al uso de internet en educación se le ha atribuido la ventaja de que las ideas presentan una gama muy amplia de contenidos, permite la participación de personas con diferentes perspectivas, crea el ciberespacio y erosiona las fronteras entre lo real y lo virtual (García, 2003). Esta autora preveía que los procesos de enseñanza y aprendizaje se podrían beneficiar de los recursos tecnológicos, pues habría un

cambio de la clase magistral y la exposición oral hacia una más autónoma [con] inclusión de personas menos o más aventajadas en el quehacer cotidiano de las clases, [cambiando hacia estudiantes más comprometidos con estas, migrando de] una evaluación de los aprendizajes basada en exámenes a una concentrada en los procesos y productos asociados, [y] de una estructura competitiva a una más cooperativa de trabajo (García, 2003, pp. 11-12).

Abogando por el uso de las TIC, Salas (2005) sostiene que la organización educativa debía crear las condiciones para su uso por cuanto las TIC tienen el potencial de elevar la capacidad comunicativa del profesorado, si bien no aporta evidencia que respalde la anterior afirmación. También se aseguraba que la tecnología digital era la solución para el desarrollo de los pueblos, pues permitía hacer mejor lo que ya se hacía y era producto de la evolución de la época industrial, de ahí que se exigía ser usuarios de las tecnologías en forma permanente, así como contar con destrezas intelectuales para comunicarse exitosamente por medio de esta (González, 2005).

Otro argumento que se ha usado para justificar el uso de las TIC en la enseñanza superior es que los egresados universitarios hallarán estas tecnologías en su vida laboral (Monge y Méndez, 2007). Asimismo, señalan los beneficios potenciales del uso de las TIC, pero tampoco aportan resultados de la evaluación del impacto de tales recursos sobre el rendimiento académico o el proceso de enseñanza.

Relacionado con el uso de las TIC e internet en educación superior, se ha desarrollado el aprendizaje colaborativo en línea, señalando que algunas de sus ventajas son posibilitar una mayor expresión, fomentar el aprendizaje constructivista, colaborar entre estudiantes y facilitadores, permitir una evaluación más formativa y eliminar las barreras espacio-temporales (Quirós, 2009). Además, se ha afirmado que los portales web de apoyo a la educación propician la autonomía y el autoaprendizaje del alumnado mejorando la enseñanza del personal docente, el aprendizaje del estudiantado y la interacción entre ambos (Salicetti y Romero, 2010). Según estos autores, el principal beneficio que percibe el alumnado con respecto al uso de la web en educación es que permite gestionar y estructurar la asignatura, por temas o semanas, así como organizar el material didáctico. Sin embargo, al igual que en los casos señalados anteriormente, los autores mencionados no cuantificaron la repercusión del uso de la plataforma web sobre el rendimiento académico.

En Costa Rica, el uso de las plataformas virtuales como apoyo a la docencia en las universidades públicas se remonta a finales de los años 90 del siglo pasado. Por ejemplo, los laboratorios virtuales en la educación a distancia se comenzaron a utilizar a partir de 1997 para cursos de ciencias biológicas (Monge y Méndez, 2007).

La División de Educología de la Universidad Nacional (UNA) inició en 2006 la incorporación de tecnologías relacionadas con el aprendizaje virtual (Ulate y Vílchez, 2010), lo que, según estas autoras, representó un gran cambio en una universidad con una fuerte tendencia hacia lo presencial y escaso uso de tecnologías para la enseñanza y aunque propusieron que el estudiantado evaluara los materiales utilizados en el aula virtual, no plantearon una evaluación de las consecuencias que esta tendría sobre el rendimiento académico.

En la UCR se han desarrollado varias experiencias con el uso de las TIC y del aula virtual. Entre ellas se mencionan las siguientes: 
- En la Facultad de Farmacia se concibió el uso de las TIC como una herramienta fundamental para la incorporación laboral, como un medio para que el cuerpo docente amplíe sus opciones y el estudiantado pueda contactar con expertos en el campo (Badilla, 2008).

- La Facultad de Odontología desarrolló una herramienta informática para funcionar como simulador y su utilidad fue bien evaluada por docentes y estudiantes (Maroto y Quirós, 2010), aunque no se cuantificó su impacto en el aprendizaje o el rendimiento académico.

- Para los cursos de Bioquímica en la Escuela de Medicina se implementó un módulo sobre bioquímica y estilos de vida saludable para utilizarlo en línea como complemento (Granados y Solano, 2010). Los autores señalan que, si bien el módulo audiovisual fue bien valorado por docentes y estudiantes, debe investigarse cómo, a partir del uso de las TIC, se aprenden conceptos específicos, se logran cambios conceptuales, se producen efectos sociocognitivos o se afecta el razonamiento complejo.

- Para la enseñanza de la Bioquímica en la Escuela de Medicina también se ha ensayado el trabajo en grupo para desarrollar un aprendizaje cooperativo en línea y se encontró una mejoría significativa en el porcentaje de aprobación cuando se utilizó el aprendizaje colaborativo; sin embargo, no investigaron en los grupos anteriores al que se evaluó ninguna de las otras variables que afectaran el rendimiento académico tales como la carga académica matriculada, la condición laboral y socioeconómica del alumnado, la calidad de la educación secundaria recibida, el interés en la carrera que se cursa y la educación de los padres (Fernández et ál., 2012).

- En 2001 se inició el Programa de Tecnologías Educativas Avanzadas, en la Facultad de Educación (Alonso, 2007). La autora describe los avances alcanzados en este campo a lo largo de unos diez años, pero no indica evaluaciones del impacto en el uso de tecnologías educativas sobre el rendimiento académico.

- En Bibliotecología y Ciencias de la Información se investigó el manejo que el estudiantado hace de la Web 2.0 a lo largo de un curso específico y se evaluó la destreza para manejar y presentar los diversos recursos disponibles en la red, así como la opinión de estos respecto a su experiencia particular; todo lo cual, en general, se consideró positivo por el autor (González, 2011).

- En Odontología (Maroto, 2011) y en Educación ambiental (Zúñiga y García, 2011) se han utilizado portafolios virtuales para evaluar aprendizajes, destrezas, habilidades, actividades y metodologías, y este se consideró como un recurso útil para estimular la reflexión y el cambio de actitud entre las personas participantes. No obstante, en el contexto de un proyecto de investigación, el portafolio busca promover la reflexión y no una calificación individual (Zúñiga y García, 2011), y demanda un seguimiento permanente e individualizado por parte del docente hacia cada estudiante. De aquí que los grupos de estudiantes en los cuales se puede aplicar esta estrategia deben ser relativamente pequeños; en los casos mencionados fueron grupos de 6 y 13 estudiantes.

- Se han aplicado las TIC en el aula para un curso de fonética francesa, al considerar que dicha herramienta contribuye a acercar a los aprendices a contextos reales de utilización de una lengua extranjera (Chao, 2014).

Como apoyo a estas experiencias, en el 2006 se creó en la UCR la Unidad de Apoyo a la Docencia Mediada con Tecnologías de la Información y de la Comunicación (METICS) y se comenzó a utilizar la plataforma Module Object-Oriented Dynamic Learning Environment (MOODLE) (Chacón, 2012). Esta autora indica que, a más de 20 años de contar con las herramientas web, se debe lograr aún un verdadero efecto en el ámbito educativo y justifica esta perentoriedad solamente con el argumento de la innovación obligatoria.

El Departamento de Bioquímica de la Escuela de Medicina en la UCR incorporó el uso del aula virtual desde hace aproximadamente diez años, como un apoyo a sus actividades docentes. Durante este tiempo dicho recurso se ha utilizado como un repositorio de las clases, materiales audiovisuales de apoyo, lecturas complementarias y actividades individuales o en grupo por medio de las cuales se busca mejorar el aprendizaje de los contenidos que se ofrecen en el aula. Aunque siempre se ha considerado que el aula virtual y las TIC 
asociadas con ella mejoran el rendimiento académico del estudiantado, no se han efectuado observaciones específicas para evaluar este efecto. Por esta razón se planteó el presente estudio utilizando la información y experiencia desarrollada con el curso de Bioquímica para Enfermería a manera de un primer análisis que, luego, pueda ampliarse con otros cursos similares.

\section{MARCo TEÓRICo}

El aula virtual se entiende como un espacio meramente conceptual, no real físicamente, utilizado como apoyo para la enseñanza (Monge y Méndez, 2007), que utiliza los recursos de las TIC, la web y las plataformas que para tal fin poseen las instituciones de educación superior tales como MOODLE y METICS en el caso de la UCR. Para los casos que se analizaron en este trabajo, el aula virtual se ha utilizado como un repositorio de información audiovisual complementaria al curso de Bioquímica para Enfermería, así como del contenido de las clases, artículos y otros materiales que el personal docente considere relevante para alcanzar los objetivos del curso; también, se ha utilizado como un medio para el desarrollo de actividades asincrónicas que incluyen tareas, foros y evaluaciones.

El rendimiento académico se valora por medio de una nota que, a su vez, depende del aprovechamiento del estudiante en las diferentes actividades (Garbanzo, 2007). Si bien el rendimiento académico se relaciona con múltiples variables cognitivas, emocionales y socioeconómicas (Garbanzo, 2007) y la aplicación de pruebas escritas elaboradas a partir de los objetivos establecidos en el programa de los cursos, no siempre es el medio idóneo para evaluar los aprendizajes (Guzmán, 2013), razones prácticas y logísticas hacen que este modo de evaluación sea el empleado en los cursos que se analizan en esta investigación.

\section{Procedimientos metodológicos}

La presente investigación se desarrolló en dos partes:

1. Análisis retrospectivo del rendimiento académico de las y los estudiantes en el curso de Bioquímica para Enfermería que impartió el Departamento de Bioquímica de la Escuela de Medicina de la UCR durante el período 2000 a 2017, en relación con el uso del aula virtual. Se seleccionó este rango de fechas para incluir un número igual de años antes y después de la incorporación del aula virtual en el curso en cuestión.

Dado que el aula virtual se comenzó a utilizar en el año 2009, se analizó el rendimiento académico correspondiente a los nueve años anteriores (2000-2008) y a los nueve siguientes (2009-2017) de que se inició el uso de dicho recurso tecnológico.

Para este análisis se consultó el archivo con las actas de las calificaciones de los cursos, cuantificando el número total de aprobados y de reprobados en cada ciclo lectivo. Los resultados se analizaron mediante una prueba de t-Student para muestras independientes, con el fin de determinar si existe una diferencia estadísticamente significativa $(\alpha=0.05)$ en el porcentaje de aprobación del curso antes y después de la incorporación del aula virtual.

2. Análisis transversal del rendimiento académico en un grupo de estudiantes del curso de Bioquímica para Enfermería en relación con la cantidad de veces con que cada estudiante accede al aula virtual.

Para este análisis se tabularon las notas obtenidas por cada alumno a lo largo del curso impartido durante agosto a diciembre de 2017, así como el número de clics que cada uno marcó en el aula virtual en el período comprendido antes de cada evaluación. El número de clics por estudiante se usó como una medida de la frecuencia con la que utilizó el aula virtual y es un dato que la plataforma Moodle genera automáticamente.

Dado que en el curso aquí analizado se ejecutaron tres exámenes parciales (A,B,C) y uno final (D), los clics por estudiante se cuantificaron en los siguientes periodos: del inicio del curso a A, de A a B, de B a C, y de $\mathrm{C}$ a D. Los resultados se analizaron mediante una prueba de correlación pareada nota-número de clics por 
examen y por nota final con un nivel de significancia de 0,05 . Todos los análisis estadísticos se prepararon empleando el programa en línea de acceso libre del Vassar College, New York (Lowry, s.f.).

\section{ANÁLISIS Y DISCUSIÓN DE RESULTADOS}

En la Tabla 1 se presenta la cantidad de estudiantes aprobados y reprobados por año en el curso de Bioquímica para Enfermería durante el período 2000 a 2017. Tomando como referencia el año 2009, en el que comenzó a utilizarse el aula virtual, el período antes indicado se puede dividir en dos lapsos iguales: del 2000 al 2008 y del 2009 al 2017. En el primero, la aprobación promedio por curso fue de $70 \%$ (DS $=15.4 \%$ ), con un promedio de estudiantes matriculados por curso de 40.2 (DS = 24.9). En el segundo, la aprobación promedio por curso fue de $59.5 \%$ (DS = 14.8\%), con un promedio de estudiantes matriculados por curso de 69.8 (DS = 29.4).

Una prueba de $\mathrm{t}$-Student permitió determinar que no existe diferencia significativa $(\mathrm{p}=0.064)$ en el promedio de aprobación por curso entre antes y después de la instauración del aula virtual. Cabe señalar que la cantidad de cursos que se ofrecieron por año calendario varió entre 1 y 5 , pues se impartieron en alguno o todos los tres ciclos lectivos que se ofrecen por año (dos ciclos regulares y uno intensivo) y en una o varias sedes de la UCR (la Sede Central y las Sedes Regionales). Por esta razón, los datos que aparecen en la Tabla 1 corresponden a la cantidad total de estudiantes en los cursos ofrecidos en los años respectivos. En la Figura 1 se presenta la tendencia histórica en el porcentaje de aprobación del curso en análisis. Puede notarse que, si bien no hubo un cambio estadísticamente significativo en el porcentaje de aprobación por curso luego de la instauración del aula virtual en el año 2009, sí se observa una tendencia histórica hacia la disminución en el parámetro indicado, tendencia sobre la cual no parece haber tenido ninguna consecuencia positiva el uso del aula virtual.

\section{TABLA 1}

Cantidad estudiantes aprobados y reprobados por año en el período 2000-2017 en el curso Bioquímica para Enfermería, Escuela de Medicina, Universidad de Costa Rica

\begin{tabular}{|c|c|c|c|c|c|}
\hline \multirow{3}{*}{ AÑO } & \multicolumn{5}{|c|}{ CANTIDAD DE ESTUDIANTES } \\
\hline & \multicolumn{2}{|c|}{ Aprobados } & \multicolumn{2}{|c|}{ Reprobados } & \multirow[b]{2}{*}{ Total } \\
\hline & $\begin{array}{l}\text { Absoluta } \\
\text { (n) }\end{array}$ & $\begin{array}{l}\text { Relativa } \\
\text { (\%) }\end{array}$ & $\begin{array}{l}\text { Absoluta } \\
\text { (n) }\end{array}$ & $\begin{array}{l}\text { Relativa } \\
(\%)\end{array}$ & \\
\hline 2000 & 26 & 66,7 & 13 & 33,3 & 39 \\
\hline 2001 & 10 & 100 & 0 & 0 & 10 \\
\hline 2002 & 58 & 69,9 & 25 & 30,1 & 83 \\
\hline 2003 & 84 & 67,2 & 41 & 32,8 & 125 \\
\hline 2004 & 95 & 76 & 30 & 24 & 125 \\
\hline 2005 & 104 & 73,8 & 37 & 26,2 & 141 \\
\hline 2006 & 58 & 58,6 & 41 & 41,4 & 99 \\
\hline 2007 & 65 & 59,6 & 44 & 40,4 & 109 \\
\hline 2008 & 76 & 66,7 & 38 & 33,3 & 114 \\
\hline 2009 & 42 & 42,4 & 57 & 57,6 & 99 \\
\hline 2010 & 90 & 84,9 & 16 & 15,1 & 106 \\
\hline 2011 & 45 & 61,6 & 28 & 38,4 & 73 \\
\hline 2012 & 50 & 69,4 & 22 & 30,6 & 72 \\
\hline 2013 & 44 & 55,7 & 35 & 44,3 & 79 \\
\hline 2014 & 52 & 55,3 & 42 & 44,7 & 94 \\
\hline 2015 & 42 & 44,7 & 52 & 55,3 & 94 \\
\hline 2016 & 56 & 54,4 & 47 & 45,6 & 103 \\
\hline 2017 & 59 & 50,4 & 58 & 49,6 & 117 \\
\hline
\end{tabular}


En la Tabla 2 se presenta el resultado del análisis de la relación entre rendimiento académico y el uso del aula virtual en un grupo de 55 estudiantes del curso de Bioquímica para Enfermería. Se observa que, si bien la media de las calificaciones en los exámenes parciales se mantuvo prácticamente constante, el uso del aula virtual mostró considerables oscilaciones promedio. Se efectuó una prueba de correlación pareada notanúmero de clics por examen y se encontró que no hubo una correlación estadísticamente significativa $(\alpha=$ $0,05)$ entre la nota de la primera evaluación y el uso del aula virtual $(\mathrm{r}=0.240, \mathrm{P}=0.077)$. A pesar de que la significancia estadística de la correlación nota-uso del aula virtual mejoró para el segundo y tercer examen parcial ( $\mathrm{r}=0.324,0.369$ y $\mathrm{P}=0.016$ y 0.005 , respectivamente), no ocurrió lo mismo para el examen final $(\mathrm{r}=0.263, \mathrm{P}=0.068)$. Por último, la correlación entre la nota final del curso y el uso del aula virtual fue significativa, aunque débil $(\mathrm{r}=0.328, \mathrm{P}=0.014)$. Tan solo entre un 5.8 y un $13.6 \%$ de la nota en cada evaluación se puede explicar por el uso del aula virtual, según lo indica el coeficiente de determinación (r2).

\section{El problema de la evaluación del rendimiento académico}

Un primer aspecto que se debe tener presente al efectuar el análisis propuesto es asociar el rendimiento académico con múltiples variables cognitivas, emocionales y socioeconómicas. Estas variables incluyen las horas asignadas al estudio, las prácticas académicas, la autoestima y competencia cognitiva del estudiante, la función del docente, los recursos didácticos disponibles, las estrategias de enseñanza, los métodos de evaluación y los factores sociodemográficos (Garbanzo, 2007, 2013, 2014). En un estudio sobre la relación entre asistencia a clase y rendimiento académico (Coello y Rizo, 2017) no se encontró una asociación significativa entre esos dos factores y se señalan otros que podrían presentar mayor incidencia tales como el trabajo del personal docente, el número de estudiantes, la mortalidad histórica del curso y las variables del contexto académico que influyen en la motivación del alumnado. 


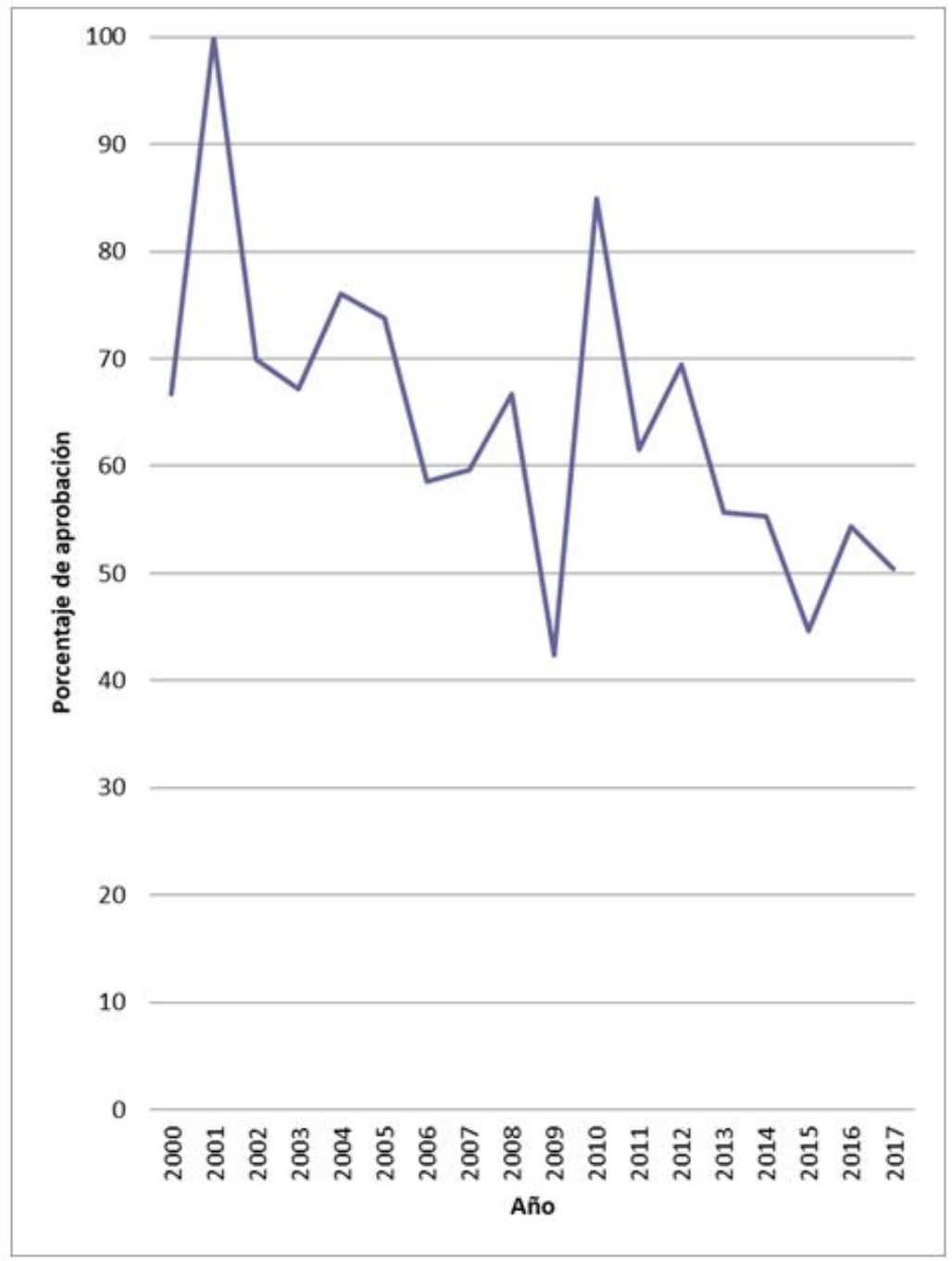

FIGURA 1

Porcentaje de aprobación por año en el curso de Bioquímica para Enfermería durante el período 2000 a 2017, Escuela de Medicina, Universidad de Costa Rica Fuente: Elaboración propia 
TABLA 2

Rendimiento académico en un grupo de 55 estudiantes del curso de Bioquímica para

Enfermería en relación con el uso del aula virtual durante el ciclo lectivo que se extendió de agosto a diciembre de 2017, Escuela de Medicina, Universidad de Costa Rica

\begin{tabular}{|c|c|c|c|c|c|}
\hline \multicolumn{2}{|c|}{$\begin{array}{l}\text { RENDIMIENTO } \\
\text { ACADÉMICO }\end{array}$} & \multirow{2}{*}{$\begin{array}{l}\text { USO DEL } \\
\text { AULA } \\
\text { VIRTUAL } \\
\text { (Cantidad de } \\
\text { clics) }\end{array}$} & \multicolumn{3}{|c|}{$\begin{array}{l}\text { ANÁLISIS DE CORRELACIÓN RENDIMIENTO } \\
\text { ACADÉMICO-USO DEL AULA VIRTUAL }\end{array}$} \\
\hline $\begin{array}{l}\text { Criterio de } \\
\text { evaluación }\end{array}$ & $\begin{array}{l}\text { Nota } \\
\text { promedio } \\
\text { porcentual }\end{array}$ & & $\begin{array}{l}\text { Coeficiente de } \\
\text { correlación (r) }\end{array}$ & $\begin{array}{l}\text { Coeficiente de } \\
\text { determinación } \\
\text { (r2) }\end{array}$ & $\mathrm{P}$ \\
\hline $\begin{array}{l}\text { Examen } \\
\text { parcial } 1\end{array}$ & $65.9(18)$ & $59.3(26)$ & 0.240 & 0.058 & 0.077 \\
\hline $\begin{array}{l}\text { Examen } \\
\text { parcial } 2\end{array}$ & $63.7(17)$ & $39.1(21)$ & 0.324 & 0.105 & 0.016 \\
\hline $\begin{array}{l}\text { Examen } \\
\text { parcial } 3\end{array}$ & $66.9(17)$ & $53.7(23)$ & 0.369 & 0.136 & 0.005 \\
\hline $\begin{array}{l}\text { Examen } \\
\text { final }\end{array}$ & $55.1(16)$ & $13.9(12)$ & 0.263 & 0.069 & 0.068 \\
\hline Nota final & $64.9(16)$ & $165(65)$ & 0.328 & 0.108 & 0.014 \\
\hline
\end{tabular}

Fuente: Elaboración propia

1. Las cifras entre paréntesis se refieren a las respectivas desviaciones estándar.

2 En Uso del aula virtual, la cantidad de clics se cuantificó en los períodos inmediatamente anteriores a la respectiva evaluación. Para Nota final se tomó la cantidad total de clics registrada durante el curso.

En el curso de Bioquímica para Enfermería en análisis, la estrategia para evaluar el rendimiento académico ha consistido, mayoritariamente y por razones prácticas, en la aplicación de pruebas escritas de selección única o múltiple. Este tipo de prueba permite examinar grupos muy numerosos, pero favorece el enfoque memorístico sobre los hechos y desvaloriza los niveles de reflexión superiores (Guzmán, 2013).

A pesar de las limitaciones anteriormente indicadas, el criterio para determinar rendimiento académico a partir de evaluaciones sumativas, principalmente mediante exámenes escritos de selección, ha resultado la estrategia más práctica para evaluar cursos como el que aquí se analiza, con contenidos abundantes y matrículas numerosas.

\section{El problema de la innovación en docencia}

Un segundo aspecto por discutir es la justificación para el uso de las TIC y del aula virtual en la docencia universitaria. La innovación como único criterio para justificar el uso de estas estrategias no parece suficiente. A menudo el término innovación es mal empleado pues, según Berkun (2010), debería utilizarse para aquellos pocos casos en los cuales se toman grandes riesgos para alcanzar cambios positivos significativos. Además, según este autor, existe el peligro de no saber en qué momento se está ante un verdadero progreso o, meramente, ante una distracción que parece representar una ganancia a corto plazo, pero que a largo plazo ocasionará consecuencias negativas. Cabría preguntarse, siguiendo a Berkun (2010), qué problema resuelve una innovación, de quién son esos problemas y cuáles nuevos crea. Estos cuestionamientos podrían aplicarse legítimamente al uso de las TIC y del aula virtual en docencia y plantearse si, lo que en su momento fue una innovación, ha venido a propiciar un cambio positivo en el aprendizaje o si, por el contrario, se ha convertido en una fuente de distracción con consecuencias negativas.

Además, si bien se señala que la experiencia de innovar es un ámbito necesario en cualquier área de trabajo, también se advierte que las propuestas de innovación educativa provienen de cómo otros conciben mejorar la práctica pedagógica, sin considerar las visiones, concepciones y creencias de quienes han obtenido éxito con las propuestas y su relación con estas características de quienes las recibirán (García, 2001). No siempre 
es posible que esa innovación propicie un cambio importante en lo que se hace porque no concuerda con la visión, concepción y creencias sobre lo que es y debería ser un espacio pedagógico. Puede ocurrir que el personal docente ejecute las directrices del sistema, pero no las crea, por lo que se convierten en elementos agregados sin contar con el aval ni ser reconocidos por el equipo de profesionales. De este modo, si se introduce un elemento ajeno al sistema, se genera una resistencia (García, 2001).

\section{Ventajas en el uso de las TIC y del aula virtual}

El uso de las TIC en la educación se ha justificado argumentando que facilitan el proceso de enseñanzaaprendizaje en el aula, puesto que las lecciones se vuelven más activas e innovadoras, mejoran la comunicación entre estudiantes y docentes, y desarrollan en el/la estudiante destrezas como el envío de información, la navegación en la red, la consulta en hipermedios, la comunicación constante y la participación activa (Gómez, 2008). Sin embargo, la autora no refiere los métodos para evaluar el desarrollo de estas destrezas ni el efecto que ellas, como medio y no como fin, puedan tener en el rendimiento académico del estudiantado.

El uso de las TIC plantea cambios civilizatorios que han transformado la forma de construir y concebir el mundo, y esto afecta a formadores y a estudiantes (Pérez y Salas, 2009). Estas autoras sostienen que por medio de herramientas digitales se "favorece el cambio de actuación y las tareas del profesorado, porque permite el diseño de ambientes de aprendizaje innovadores centrados en el alumno y no en el docente” (Pérez y Salas, 2009, p. 4). Señalan "datos empíricos que muestran que los estudiantes con acceso a esas herramientas realizan sus estudios en poco tiempo, requieren menos recursos y se gradúan mejor preparados para enfrentar el mercado laboral" (Pérez y Salas, 2009, p. 11). Además, afirman que estas tecnologías

son medios de comunicación poderosos que permiten el quehacer individual y potencian el trabajo en equipo, el aprendizaje colaborativo y la investigación interdisciplinaria, y conducen a un salto cualitativo en la enseñanza que afecta la entrega de la docencia, porque permite acceder, producir y compartir materiales educativos de diversa naturaleza en forma barata y estéticamente más rica (Pérez y Salas, 2009, p. 18).

Charpentier (2014) evaluó, mediante una consulta vía correo electrónico, la frecuencia con la cual el alumnado de inglés en la UCR utilizaron las TIC y sus impresiones al respecto, y encontraron que creen que la tecnología es una ayuda efectiva para la enseñanza. Sostiene que la tecnología ayuda a que el aprendizaje del lenguaje sea más rápido, está disponible cuando no están las y los docentes y ofrece una atención individualizada. No obstante, a pesar de estos potenciales beneficios, el estudio en mención no midió el efecto del uso del aula virtual sobre el rendimiento académico.

\section{Desventajas en el uso de las TIC y del aula virtual}

Puig (2016) advierte que uno de los inconvenientes que acarrea el uso de internet para la educación es que, de alguna manera, todo es válido, y emerge la figura informe de un autor global que lo dice todo y, a la vez, no dice absolutamente nada.

Uno de los reparos que se plantea al uso de las TIC en docencia es que se trata de herramientas eficaces para aprender a manipular software $\mathrm{u}$ otras aplicaciones tecnológicas, pero no son instrumentos que desarrollen las habilidades cognitivas superiores (Brown, 2005). El autor plantea algunas acciones para mejorar el uso de las TIC en la docencia, entre las cuales destaca la investigación para dar a conocer las consecuencias de las iniciativas para incorporar las TIC y sus resultados en la enseñanza y en el aprendizaje. "La investigación es importante para dotar al discurso de la incorporación de las TIC en la docencia de argumentos con sustento objetivo y científico" (Brown, 2005, p. 18).

Quirós (2009) ha señalado algunos factores negativos del aprendizaje colaborativo en línea tales como la pérdida del contacto humano, la reducida accesibilidad a internet o la posibilidad de convertirse en 
distractores de aprendizaje. Señala la necesidad de que el docente esté en renovación constante y la obligación de revolucionar la educación, aunque parece enfatizar en la renovación por sí misma más que en el mejoramiento del aprendizaje.

Para Chao (2014) las TIC "producen un cambio en el proceso formativo al dejar de ser este una recepción y memorización de datos recibidos en clase, para convertirse en una búsqueda permanente, análisis y reelaboración de informaciones obtenidas en la red" (p. 4). El mismo autor sostiene que algunas de sus desventajas son: generar distracción y dispersión en el estudiante, difundir información no fiable, dar aprendizajes incompletos y superficiales, propiciar la pereza y causar aislamiento y ansiedad. Por ello sugiere que, para evitar estos riesgos, las TIC deben integrarse en un marco de acción pedagógico con un objetivo específico y no porque sea una moda o una exigencia.

Se ha estudiado el papel de las TIC en el contexto de la educación a distancia (Vargas y Villalobos, 2018) notándose que la tecnología en sí misma no marca una diferencia en la mejora del proceso de aprendizaje, sino que debe ser mediada de tal modo que sea útil como herramienta para tal fin. Salas (2005) advierte que si las TIC se utilizan al estilo tradicional de los textos escritos, probablemente su efecto sea menor que si se utilizan como multimedios.

Carr (2010) apunta que internet involucra todos los sentidos, con excepción del olfato y el gusto, y lo hace simultáneamente dirigiendo la atención con una insistencia mayor de lo que nunca lo han hecho la radio, la televisión o los periódicos. También advierte que la necesidad de evaluar los enlaces y ejecutar elecciones de navegación relacionadas, mientras también se procesa una multiplicidad de estímulos sensoriales fugaces, requiere una coordinación mental constante y la toma de decisiones que distraen el cerebro del trabajo para interpretar un texto u otra información. Para dicho autor, aunque muchos educadores creyeron que la introducción de hiperenlaces en los textos desplegados en la pantalla de una computadora sería una bendición para el aprendizaje fortaleciendo el pensamiento crítico del estudiantado, las investigaciones continúan mostrando que las personas lectoras de textos lineales comprenden, recuerdan y aprenden más que aquellas que lo hacen en uno salpicado de enlaces. El medio utilizado para presentar las palabras oscurece su significado y la evidencia preponderante indica que las demandas incrementadas de una toma de decisión y un procesamiento visual en el hipertexto desacopla el desempeño de la lectura, particularmente cuando se compara con una presentación lineal tradicional. Muchos educadores también asumen que el multimedia mejorará la comprensión y fortalecerá el aprendizaje, pero esta presunción, por mucho tiempo aceptada sin fuerte evidencia, también ha sido contradicha por las investigaciones (Carr, 2010). Las frecuentes interrupciones en internet dispersan los pensamientos, debilitan la memoria e incrementan la tensión y la ansiedad.

Si bien algunas habilidades cognitivas se fortalecen con el uso de las computadoras e internet, estas tienden a involucrar funciones mentales de bajo nivel o más primitivas tales como la coordinación manoojo, respuestas reflejas y el procesamiento de información visual. Además, se ha observado que cuanto más multitarea sea una persona, menos deliberativa se vuelve, es decir, es menos hábil para pensar y razonar sobre un problema (Carr, 2010). Como evidencia de lo anterior, Carr señala que los puntajes en los exámenes

PSAT, que se aplican a estudiantes de secundaria en Estados Unidos, no se incrementaron durante el periodo de 1999 a 2008, años en los que el uso de internet en hogares y colegios se expandió dramáticamente.

\section{Una estrategia integradora}

Del análisis anterior cabe plantearse la necesidad de que el personal docente, además de incorporar los recursos tecnológicos, valoren permanentemente "el objetivo que subyace a las situaciones de aprendizaje, actividades y tareas teniendo presente que, si lo virtual es aquello que posee las mismas características y efectos que los objetos o situaciones reales que presenta" (García, 2003, pp. 13, 18), la virtualidad en educación 
debería permitir que el individuo se construya mediante las interacciones con otras personas y con los objetos (García, 2003).

Más que el uso del aula virtual o las TIC asociadas, el enfoque curricular basado en competencias y resultados de aprendizaje debería superar al que se basa "en la mera transmisión de conocimientos y avanzar hacia el desarrollo de capacidades, habilidades, destrezas y actitudes que permitan disponer de múltiples recursos para actuar competentemente" (García, Fonseca y Concha, 2015, p. 2).

En ese sentido, el sistema educativo debería promover, impulsar y orientar "al estudiante en la adquisición de herramientas que le permitan aprender más y con calidad” (García et ál., 2015, p. 9), haciendo que el aprendizaje dependa "de lo que la persona haga, es decir, de los procesos cognitivos que ponga en marcha al aprender y, por lo tanto, de las estrategias que desarrollan esos procesos" tales como adquisición, codificación, recuperación y de apoyo (García et ál., 2015, p. 9).

Para el desarrollo de estas destrezas debería considerarse la posibilidad de recuperar el uso más frecuente de los libros de texto impresos, así como la ejecución de actividades en el aula las cuales incentiven en el estudiantado sus capacidades personales como la comunicación, organización, planificación y responsabilidad.

\section{Conclusiones}

1. La incorporación del aula virtual en el curso de Bioquímica para Enfermería de la UCR en el año 2009 no produjo un cambio estadísticamente significativo en el porcentaje de aprobación por curso ni en la tendencia histórica hacia su disminución.

2. En el curso ofrecido durante el segundo ciclo lectivo de 2017 la correlación entre el uso del aula virtual y las notas de cada examen no fue estadísticamente significativa o fue débil.

3. Si bien el rendimiento académico estudiantil es un resultado multifactorial, el uso del aula virtual en el curso que se analizó permitió explicar tan solo una mínima parte de tal fenómeno.

4. Debe analizarse si el uso de las TIC y del aula virtual en docencia ha venido a propiciar un cambio positivo en el aprendizaje o si, por el contrario, se ha convertido en una fuente de distracción con consecuencias negativas.

5. Aunque el uso de las tecnologías en educación es ventajoso en el aprendizaje de ciertas destrezas, la evaluación de su efecto sobre el rendimiento académico no se ha llevado a cabo de un modo preciso y objetivo.

6. El uso de aulas virtuales con sus diversos recursos audiovisuales e hipertextos podrían representar un factor de distracción que reduzca la comprensión y el aprendizaje, comparado con aquellas personas que practican lecturas lineales de textos impresos.

7. El uso más frecuente de los libros de texto impresos, así como la ejecución de actividades en el aula que incentiven en el estudiantado sus capacidades personales deberían mejorar su rendimiento académico como alternativa prioritaria al uso del aula virtual o las TIC.

\section{REFERENCIAS}

Alonso, J. (2007). Programa de tecnologías educativas avanzadas: una reseña histórica. Actualidades Investigativas en Educación, 7,1-12. https://doi.org/10.15517/aie.v7i4.9300

Badilla, B. (2008). Foro con experto. Aplicación de las nuevas tecnologías de la información y comunicación en la formación farmacéutica. Actualidades Investigativas en Educación, 8(2), 1-19. https://doi.org/10.15517/aie.v8 i2.9330

Berkun, S. (2010). The Myths of Innovation. Sebastopol, California: O’Reilly. 
Brown, J. (2005). Incorporación de las tecnologías de información y comunicación en la docencia universitaria estatal costarricense: problemas y soluciones. Actualidades Investigativas en Educación, 5(1), 1-21. https://doi.org/10 $.15517 /$ aie.v5i1.9118

Carr, N. (2010). The Shallows. What the internet is doing to our brains. New York: W.W. Norton.

Carvajal, Á. (2002). Informática educativa: una reflexión crítica. Actualidades Investigativas en Educación, 2(1), 1-21. https://doi.org/10.15517/aie.v2i1.8466

Chacón, S. (2012). El acompañamiento docente: el quehacer de la unidad METICS. Actualidades Investigativas en Educación, 12(1), 1-24. https://doi.org/10.15517/aie.v12i1.10258

Chao, K. (2014). Estrategias didácticas mediadas con TIC en un curso de expresión oral francesa. Actualidades Investigativas en Educación, 14(2), 1-30. https://doi.org/10.15517/aie.v14i2.14801

Charpentier, W. (2014). The use of ICTs in the BA in english teaching. Actualidades Investigativas en Educación, 14(1), 1-23. https://doi.org/10.15517/aie.v14i1.13211

Coello, J. y Rizo, F. (2017). Rendimiento académico universitario y asistencia a clases: Una visión. Revista Educación, 41(2), 1-17. https://doi.org/10.15517/revedu.v41i2.18477

Fernández, M., Alape, A., Artolozaga, M., Calvo, L., Centeno, C., Gómez, G., Granados, K. (2012). Aprendizaje cooperativo en un curso de bioquímica: opinión de estudiantes y efecto en su rendimiento académico. Actualidades Investigativas en Educación, 12(1), 1-26. https://doi.org/10.15517/aie.v12i1.10254

Garbanzo, G. (2007). Factores asociados al rendimiento académico en estudiantes universitarios, una reflexión desde la calidad de la educación superior pública. Revista Educación, 31(1), 43-63. https://doi.org/10.15517/revedu .v31i1.1252

Garbanzo, G. (2013). Factores asociados al rendimiento académico en estudiantes universitarios desde el nivel socioeconómico: Un estudio en la Universidad de Costa Rica. Revista Electrónica Educare, 17(3), 57-87. Recuperado de: http://www.revistas.una.ac.cr/index.php/EDUCARE/article/view/5258/5069

Garbanzo, G. (2014). Factores asociados al rendimiento académico tomando en cuenta el nivel socioeconómico: Estudio de regresión múltiple en estudianes universitarios. Revista Electrónica Educare, 18(1), 119-154. Recuperado de: http://www.revistas.una.ac.cr/index.php/EDUCARE/article/view/5566

García, F., Fonseca, G., y Concha, L. (2015). Aprendizaje y Rendimiento Académico en Educación Superior: Un Estudio Comparado. Actualidades Investigativas en Educación, 15(3), 404-429. https://doi.org/10.15517/aie.v $15 \mathrm{i} 3.21072$

García, J. (2001). Supuestos epistemológicos que subyacen a la innovación educativa. Actualidades Investigativas en Educación, 1(1), 1-5. https://doi.org/10.15517/aie.v1i1.8457

García, J. (2003). El potencial tecnológico y el ambiente de aprendizaje con recursos tecnológicos: informáticos, comunicativos y de multimedia. Una reflexión epistemológica y pedagógica. Actualidades Investigativas En Educación, 3(1), 1-23. https://doi.org/10.15517/aie.v3i1.9009

Gómez, G. (2008). El uso de la tecnología de la información y la comunicación y el diseño curricular. Revista Educación, 32(1), 77-97. https://doi.org/10.15517/revedu.v32i1.525

González, E. (2011). Recursos de Google para el desarrollo de una unidad didáctica con estudiantes de educación superior. Actualidades Investigativas En Educación, 11, 1-15. https://doi.org/10.15517/aie.v11i4.10228

González, V. (2005). Tecnología digital: reflexiones pedagógicas y socioculturales. Actualidades Investigativas en Educación, 5(1), 1-24. https://doi.org/10.15517/aie.v5i1.9121

Granados, J. y Solano, A. (2010). Desarrollo de un módulo para enseñanza del metabolismo y estilos de vida saludable en estudiantes universitarios. Actualidades Investigativas En Educación, 10(3), 1-27. https://doi.org/10.15517 /aie.v10i3.10146

Gurdián, A. (2001). Las nuevas tecnologías de la información y de la comunicación (NTIC) en la educación universitaria. Actualidades Investigativas en Educación, 1(1), 1-6. https://doi.org/10.15517/aie.v1i1.8459 
Guzmán, P. (2013). La evaluación de los aprendizajes en la sección de química general de la Escuela de Química, de la Universidad de Costa Rica. Actualidades Investigativas en Educación, 13(3), 1-28. https://doi.org/10.15517 /aie.v13i3.12038

Hernández, A. (2002). Los desafíos de la docencia universitaria. Revista Educación, 26(2), 117-124. https://doi.org/ $10.15517 /$ revedu.v26i2.2907

Hernández, J. (2002). Usos de la tecnología en educación. Educare, (2), 29-33. Recuperado de https://bit.ly/30JnDBF

Lowry, R. (s.f.). VassarStats: Web Site for Statistical Computation. Recuperado de http://www.vassarstats.net/textbo ok/index.html

Luján, M. y Salas, F. (2009). Enfoques teóricos y definiciones de la tecnología educativa en el siglo XX. Actualidades Investigativas en Educación, 9(2), 1-29. https://doi.org/10.15517/v9i2.9545

Maroto, O. (2011). El portafolio digital utilizado en la evaluación de la Clínica de Odontología en la Universidad de Costa Rica (UCR): reporte de una experiencia. Actualidades Investigativas en Educación, 11(4), 1-21. https:// doi.org/10.15517/aie.v11i4.10234

Maroto, O. y Quirós, M. (2010). Uso de un medio tecnológico educativo en educación superior como un recurso didáctico: reporte de una experiencia en Odontología. Actualizaciones Investigativas en Educación, 10(2), 1-21. https://doi.org/10.15517/aie.v10i2.10122

Monge, J. y Méndez, V. (2007). Ventajas y desventajas de usar laboratorios virtuales en educación a distancia: la opinión del estudiantado en un proyecto de seis años de duración. Revista Educación, 31(1), 91-108. https://doi.org/1 $0.15517 /$ revedu.v31i1.1255

Pérez, B. y Salas, F. (2009). Hallazgos en investigación sobre el profesorado universitario y la integración de las TIC en la enseñanza. Actualidades Investigativas en Educación, 9(1), 1-25. https://doi.org/10.15517/aie.v9i1.9381

Puig, E. (2016). La gran adicción (Kindle iOs). Barcelona: Arpa y Alfil.

Quirós, E. (2009). Recursos didácticos digitales: medios innovadores para el trabajo colaborativo en línea. Revista Electrónica Educare, 53(2), 47-62. Recuperado de: https://bit.ly/30FR4V4

Regueyra, M. (2011). Aprendiendo con las TIC: una experiencia universitaria. Actualidades Investigativas en Educación, 11, 1-29. Recuperado de https://revistas.ucr.ac.cr/index.php/aie/article/view/10230

Rivera, L., Fernández, K., Guzmán, F. y Eduardo, J. (2017). La aceptación de las TIC por profesorado universitario: Conocimiento, actitud y practicidad. Revista Electrónica Educare, 21(3), 1-18. Recuperado de: http://www.rev istas.una.ac.cr/index.php/EDUCARE/article/view/7727/11483

Salas, F. (2002). Epistemología, educación y tecnología educativa. Revista Educación, 26(1), 9-18. https://doi.org/10 $.15517 /$ revedu.v26i1.2873

Salas, F. (2005). Hallazgos de la investigación sobre la inserción de las tecnologías de la información y la comunicación (TIC) en la enseñanza: la experiencia de los últimos diez años en los Estados Unidos. Revista Educación, 29(2), 53-66. https://doi.org/10.15517/revedu.v29i2.2239

Salicetti, A. y Romero, C. (2010). La plataforma de apoyo a la docencia como opción metodológica para el aprendizaje de competencias. Revista Educación, 34(1), 83-100. https://doi.org/10.15517/revedu.v34i1.499

Troncoso, O., Cuicas, M. y Debel, E. (2010). El modelo b-learning aplicado a la enseñanza del curso de matemática en la carrera de ingeniería civil. Actualidades Investigativas En Educación, 10(3), 1-28. https://doi.org/10.1551 7/aie.v10i3.10151

Ulate, G. y Vílchez, E. (2010). Una retrospección y visión de futuro sobre el uso e implementación de las tecnologías de la información y la comunicación, para el aprendizaje virtual en el contexto de la División de Educología de la Universidad Nacional de Costa Rica. Revista Electrónica Educare, 14(1), 19-36. Recuperado de http://www. revistas.una.ac.cr/index.php/EDUCARE/article/view/1506

Vargas, A. y Villalobos, G. (2018). El uso de plataformas virtuales y su impacto en el proceso de aprendizaje en las asignaturas de las carreras de Criminología y Ciencias Policiales, de la Universidad Estatal a Distancia de Costa Rica. Educare, 22(1), 1-20. https://doi.org/10.15359/ree.22-1.2 
Zúñiga, C. y García, J. (2011). Uso de un portafolio virtual para incorporar la dimensión ambiental: Una experiencia de investigación-acción en el aula. Actualidades Investigativas En Educación, 11, 1-29. https://doi.org/10.1551 7/aie.v11i4.10227

\section{Notas}

1. Las cifras entre paréntesis se refieren a las respectivas desviaciones estándar.

2. En Uso del aula virtual, la cantidad de clics se cuantificó en los períodos inmediatamente anteriores a la respectiva evaluación. Para Nota final se tomó la cantidad total de clics registrada durante el curso. Fuente: Elaboración propia

1. La incorporación del aula virtual en el curso de Bioquímica para Enfermería de la UCR en el año 2009 no produjo un cambio estadísticamente significativo en el porcentaje de aprobación por curso ni en la tendencia histórica hacia su disminución.

2. En el curso ofrecido durante el segundo ciclo lectivo de 2017 la correlación entre el uso del aula virtual y las notas de cada examen no fue estadísticamente significativa o fue débil.

3. Si bien el rendimiento académico estudiantil es un resultado multifactorial, el uso del aula virtual en el curso que se analizó permitió explicar tan solo una mínima parte de tal fenómeno.

4. Debe analizarse si el uso de las TIC y del aula virtual en docencia ha venido a propiciar un cambio positivo en el aprendizaje o si, por el contrario, se ha convertido en una fuente de distracción con consecuencias negativas.

5. Aunque el uso de las tecnologías en educación es ventajoso en el aprendizaje de ciertas destrezas, la evaluación de su efecto sobre el rendimiento académico no se ha llevado a cabo de un modo preciso y objetivo.

6. El uso de aulas virtuales con sus diversos recursos audiovisuales e hipertextos podrían representar un factor de distracción que reduzca la comprensión y el aprendizaje, comparado con aquellas personas que practican lecturas lineales de textos impresos.

7. El uso más frecuente de los libros de texto impresos, así como la ejecución de actividades en el aula que incentiven en el estudiantado sus capacidades personales deberían mejorar su rendimiento académico como alternativa prioritaria al uso del aula virtual o las TIC.

\section{BY-NC-ND}

